Europhysics Letters

PREPRINT

\title{
Fermionic Casimir effect in case of Andreev reflection
}

\author{
A. Bulgac ${ }^{1}$, P. Magierski ${ }^{1,2}$ and A. Wirzba ${ }^{3}$ \\ 1 Department of Physics, University of Washington - Seattle, WA 98195-1560, USA \\ 2 Faculty of Physics, Warsaw University of Technology - ul. Koszykowa 75, 00-662 \\ Warsaw, Poland \\ 3 Institut für Kernphysik, Forschungszentrum Jülich - D-52425 Jülich, Germany
}

PACS. 03.65.Sq - Semiclassical theories and applications.

PACS. 74.45.+c - Proximity effects; Andreev effect; SN and SNS junctions.

PACS. 74.81.-g - Inhomogeneous superconductors and superconducting systems.

\begin{abstract}
We describe the Fermionic Casimir effect in the case of two spherical superfluid scatterers immersed in a normal Fermi system. It is shown that due to the focusing property of Andreev reflection this new Casimir-like energy is significantly enhanced when compared to the case of normal scatterers with specular reflection.
\end{abstract}

Introduction. - If two or more voids (or hard-wall scatterers) are formed in a normal uniform Fermi system, effective Casimir-like interactions between such voids appear [1,2]. For example, two spherical voids of radius $a$ with distance between their centers $R$ in a Fermi medium at zero temperature and with Fermi wave vector $k_{\mathrm{F}}$ would interact with each other with the effective potential energy

$$
E_{\mathrm{C}}(R) \simeq-\nu_{\mathrm{deg}} \frac{\hbar^{2} k_{\mathrm{F}}^{2}}{2 m} \frac{a^{2}}{2 \pi R(R-2 a)} j_{1}\left[2 k_{\mathrm{F}}(R-2 a)\right]
$$

where $\nu_{\mathrm{deg}}$ is the spin-isospin degeneracy factor. Here $j_{1}(x)$ is the spherical Bessel function and $m$ the fermion mass. There is an illuminating explanation and easy derivation of this expression based on semi-classical physics — periodic orbits theory in particular. One can show that the energy density of an infinite and otherwise homogeneous Fermi system is affected by the presence of the two spherical voids and that the main contribution to this energy density change is determined by the "almost standing wave" created between the two scatterers. As was shown in refs. [1,2], this argument can be extended, in principle, to an arbitrary number of scatterers of arbitrary shapes. Volovik has arrived at similar conclusions by studying 1dimensional systems [3] and he refers to this energy as the Mesoscopic Casimir energy or force. In his discussion of this new type of Casimir energy, Volovik stresses that this energy is governed by the "microscopic trans-Planckian physics," unlike the usual Casimir energy, which is determined by the long-wave physics.

The Fermionic Casimir energy appears because of the Dirichlet boundary conditions at the voids, which semi-classically correspond to specular reflections. If, however, the boundary separates a normal Fermi system from a superfluid Fermi system, the specular reflection is

(C) EDP Sciences 


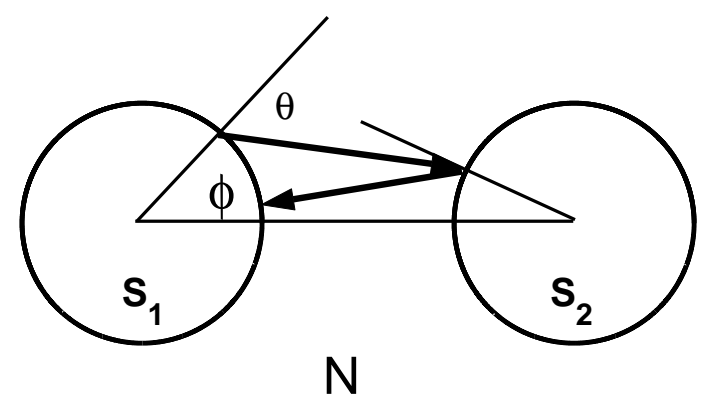

Fig. 1 - Two superfluid grains $\mathrm{S}_{1}$ and $\mathrm{S}_{2}$ immersed in a normal Fermi gas $\mathrm{N}, \phi$ is the angle at center specifying the position of the emerging ray on the first disk/sphere and $\theta$ the angle made by its momentum with the normal.

replaced with Andreev reflection [4]. A particle at the Fermi energy in the normal part of the Fermi system, impinging on the boundary between the normal and superfluid system, is retro-reflected as a hole, irrespective of the shape of the boundary. This rather simple but amazing fact has apparently quite unexpected consequences. Let us imagine now that we have two spherical superfluid grains embedded in a normal Fermi system. One might then naively expect that there is an infinite number of stable (classical) periodic trajectories between two such objects. This situation has to be contrasted with the case of specular reflection, when only one unstable trajectory between the "noses" of the two spheres exist. The latter trajectory is responsible for the change in the density of states, which ultimately results in the Fermionic Casimir energy discussed in refs. [1-3]. One can now expect that an infinite number of stable periodic trajectories would lead to a very large change in the density of states if two superfluid grains are embedded into a normal Fermi system. From this point of view the fact that two spherical superfluid scatterers do not generate a chaotic dynamics is initially quite a surprise. Our goal here is to determine the character and magnitude of the Fermionic Casimir energy in the case of two spherical superfluid scatterers embedded in a normal Fermi system. The result we obtain can easily be generalized to scatterers of arbitrary shapes and to any number of scatterers in a leading order approximation, which we shall define more carefully below. We would like to mention here that even though other aspects of the physics of superfluid grains of various sizes embedded in a normal Fermi system have been addressed before (see refs. [5,6] and references therein), the existence and nature of the Fermionic Casimir energy was never considered. Various other aspects of the quantum chaos in so-called Andreev billiards/stadiums have been considered by many authors [7], but spatial configurations of the type we shall study here have not. Our interest stems partially from the physics of neutron stars, where such structures are expected to exist $[1,8]$. Moreover, the possible relevance of Andreev reflection was mentioned in connection with the appearance of QCD-color-superfluid "grains" in the deconfined quark-gluon phase in the core of neutron [9] or of quark stars, and also in connection with the observed cosmological asymmetry between matter and anti-matter and the origin of dark matter [10].

Two superfluid grains. - To set the stage, let us consider the geometry illustrated in fig.1] In the Andreev approximation any straight line joining two arbitrary points on the surface of the two superfluid grains leads to a classical periodic orbit. A particle (p) leaving the surface of one grain, hits the second grain somewhere and it is reflected exactly backward as a hole (h) and it thus returns to the initial point on the first grain, where it now transforms, after 


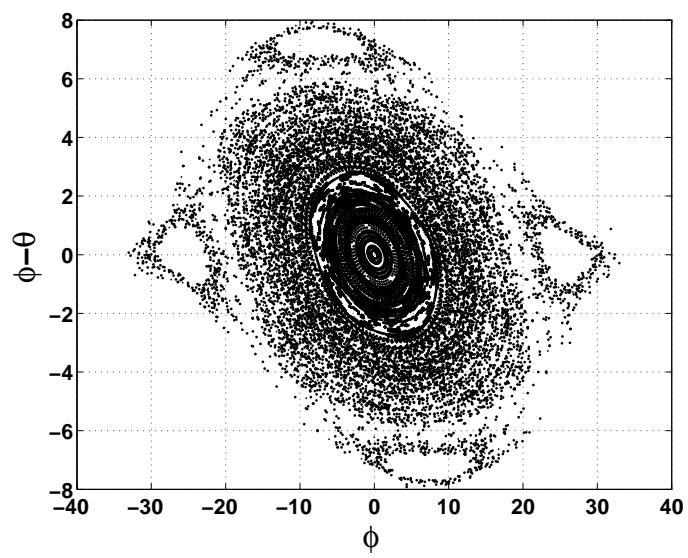

Fig. 2 - The Poincaré surface of section for the case of two superfluid circular/spherical grains embedded in a normal Fermi gas (in two dimensions), separated by a distance four times larger then their respective radii $(R=6 a)$. The ratio of the particle and hole momenta is here $k_{\mathrm{p}} / k_{\mathrm{h}}=1.5$. Only stable/elliptic trajectories parameterized by the angle $\phi$ (in degrees), where the particle originates on the surface of the disk $\mathrm{S}_{1}$, and by the angle $\phi-\theta$ (in degrees) between the momentum of the particle and a parallel of the center-to-center axis are shown. The relatively small values of $\phi-\theta$ show that most trajectories run in a tube around the line joining the two centers.

another Andreev reflection, into a particle. This is strictly correct if the particle has exactly the Fermi energy. Then the energy of both particle and hole states are equal. Otherwise the relation between the angles of the incoming particle/hole and the reflected hole/particle resembles Snell's law:

$$
k_{\mathrm{p}} \sin \theta_{\mathrm{p}}=k_{\mathrm{h}} \sin \theta_{\mathrm{h}}, \quad \frac{k_{\mathrm{p}}^{2}}{2}+\frac{k_{\mathrm{h}}^{2}}{2}=2 \mu=k_{\mathrm{F}}^{2}
$$

where $\mu$ is the Fermi energy and $k_{p}>k_{\mathrm{F}}, k_{\mathrm{h}}<k_{\mathrm{F}}$ denote the fermion momentum in a particle state and a hole state, respectively (we shall use units: $\hbar^{2}=m=1$ ). Note that the energies of the particle and hole states are symmetric with respect to the Fermi energy. Moreover, the incident and reflected rays are on the same side of the normal to the surface at the point of incidence, as opposed to the case of specular reflection. Consequently, one can show that, unlike in the case of normal reflection, the disks/spheres now have a focusing effect.

Instead of trying to seek various possible periodic trajectories we simply generated a Poincaré surface of section. Only those orbits are stable for which the separation between the centers of the grains satisfies the following constraint, derived by requiring that the eigenvalues of the monodromy matrix have to be inside or on the unit circle in the complex plane:

$$
\frac{R}{2 a} \leq \frac{k_{\mathrm{p}}}{k_{\mathrm{p}}-k_{\mathrm{h}}},
$$

where we have assumed that $\left|k_{p}-k_{h}\right| / k_{F} \ll 1$. If this constraint is not satisfied the orbits have a hyperbolic/unstable character. In the case of equality the eigenvalues of the monodromy matrix are equal to (minus) one, the orbits are parabolic/marginally stable in character and elliptic/stable otherwise.

Exact expression of the density of states. - The structure of the classical phase space briefly described here would apparently imply a rather complex quantum mechanical dynam- 
ics. As we shall show, however, the most important features of the density of states in such systems can be understood and described quite accurately in terms of a simplest periodic orbit and its repetitions. In order to determine the change in the local density of states we shall construct explicitly the scattering states and determine the phase shifts by extending the formalism used in case of normal systems $[2,11,12]$ to Bogoliubov-de Gennes equations,

$$
\left(\begin{array}{cc}
\mathbf{T}-\mu & \Delta(\boldsymbol{r}) \\
\Delta^{*}(\boldsymbol{r}) & -(\mathrm{T}-\mu)
\end{array}\right)\left(\begin{array}{c}
u(\boldsymbol{r}) \\
v(\boldsymbol{r})
\end{array}\right)=E\left(\begin{array}{c}
u(\boldsymbol{r}) \\
v(\boldsymbol{r})
\end{array}\right)
$$

where $\mathrm{T}$ is the kinetic energy operator, $E$ is the quasi-particle energy, $u(\boldsymbol{r})$ and $v(\boldsymbol{r})$ are the particle and hole spatial components of the quasi-particle wave function and $\Delta(\boldsymbol{r})$ is the pairing field, assumed to be constant inside the two scatterers, i.e. $|\Delta(\boldsymbol{r})|=\Delta$, and vanishing outside. For the sake of simplicity of the analysis we assume that the mean field vanishes everywhere and that the two scatterers have the same radius $a$. Physically it makes sense to discuss many fermion systems for which $k_{\mathrm{F}} a \gg 1$. One can distinguish further between small and large superfluid grains, depending on whether the coherence length $\xi=k_{\mathrm{F}} / \pi \Delta$ is smaller or larger than the radius of the grain $a$. The pairing fields in the two grains need not to have the same phase, which for the sake of simplicity of the argument we consider to be constant over the grain. As in ref. [2], we shall use Krein's formula [13], generalized to the case of Bogoliubov-de Genes equations. Krein's formula expresses the change in the density of states through the phase shifts, and represents a generalization of a much simpler formula due to Beth and Uhlenbeck [14]. We thus obtain in the 2-dimensional case (the 3-dimensional case has a very similar structure):

$$
\begin{aligned}
& \delta g(E)=\frac{\mathrm{d} \delta N(E)}{\mathrm{d} E}, \quad \delta N(E)=-\frac{1}{\pi} \operatorname{Im} \ln \operatorname{det}(\mathrm{M}), \\
& \mathrm{M}=\left(\begin{array}{cccc}
\ldots & \ldots & \ldots & \ldots \\
\ldots & \mathrm{M}_{m m^{\prime}} & \mathrm{M}_{m m^{\prime}+1} & \ldots \\
\ldots & \mathrm{M}_{m+1 m^{\prime}} & \mathrm{M}_{m+1 m^{\prime}+1} & \ldots \\
\ldots & \ldots & \cdots & \ldots
\end{array}\right) \\
& \mathrm{M}_{m m^{\prime}}=\left(\begin{array}{cc}
\mathbb{1} & (-1)^{m+1} \mathrm{~A}_{m m^{\prime}} \\
(-1)^{m+1} \mathrm{~B}_{m m^{\prime}} & \mathbb{1}
\end{array}\right) \text {, } \\
& \mathrm{A}_{m m^{\prime}}=\left(\begin{array}{cc}
t_{\mathrm{pp}}^{m}(a, E, \mu, \Delta) H_{m-m^{\prime}}^{(1)}\left(k_{\mathrm{p}} R\right) & t_{\mathrm{ph}}^{m}(a, E, \mu, \Delta) H_{m-m^{\prime}}^{(2)}\left(k_{\mathrm{h}} R\right) \\
t_{\mathrm{hp}}^{m}(a, E, \mu, \Delta) H_{m-m^{\prime}}^{(1)}\left(k_{\mathrm{p}} R\right) & t_{\mathrm{hh}}^{m}(a, E, \mu, \Delta) H_{m-m^{\prime}}^{(2)}\left(k_{\mathrm{h}} R\right)
\end{array}\right), \\
& \mathrm{B}_{m m^{\prime}}=\left(\begin{array}{cc}
t_{\mathrm{pp}}^{m}(a, E, \mu, \Delta) H_{m-m^{\prime}}^{(1)}\left(k_{\mathrm{p}} R\right) & t_{\mathrm{ph}}^{m}(a, E, \mu, \Delta) e^{i \phi_{\Delta}} H_{m-m^{\prime}}^{(2)}\left(k_{\mathrm{h}} R\right) \\
t_{\mathrm{hp}}^{m}(a, E, \mu, \Delta) e^{-i \phi_{\Delta}} H_{m-m^{\prime}}^{(1)}\left(k_{\mathrm{p}} R\right) & t_{\mathrm{hh}}^{m}(a, E, \mu, \Delta) H_{m-m^{\prime}}^{(2)}\left(k_{\mathrm{h}} R\right)
\end{array}\right),
\end{aligned}
$$

with $k_{\mathrm{p}}^{2}=2(\mu+E), k_{\mathrm{h}}^{2}=2(\mu-E)$ and where $m, m^{\prime}=0, \pm 1, \pm 2, \cdots$ are angular momenta. The angle $\phi_{\Delta}$ is the relative phase of the pairing fields in the two grains and $\mathbb{1}$ is the identity matrix. The matrix elements $t_{\mathrm{pp}}^{m} \equiv t_{\mathrm{pp}}^{m}(a, E, \mu, \Delta), \ldots$ are equal to half of the $T$-matrix elements for the scattering of a particle/hole of angular momentum $m$ off a single spherical superfluid grain (centered at the origin of the coordinate system), and the ordinary Hankel functions $H_{m-m^{\prime}}^{(1)}\left(k_{\mathrm{p}} R\right), \ldots$ determine the propagation between the grains.

Asymptotic approximations. - Even though these formulas are already suitable to perform explicit calculations, one can take advantage of the fact that $k_{\mathrm{F}} R \gg 1$ and obtain significantly simpler expressions by retaining only the leading term in an asymptotic expansion of the corresponding Hankel functions. One thus arrives at the following much more 


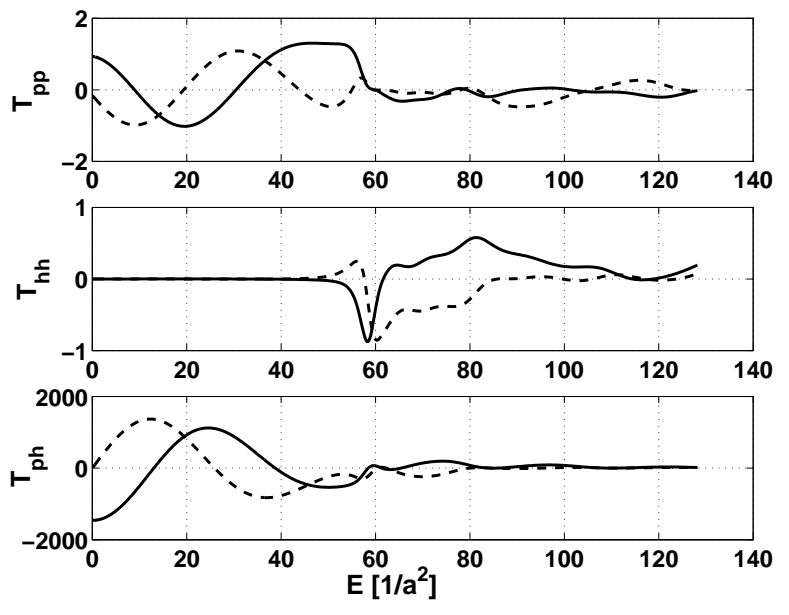

Fig. 3 - The structure functions $T_{\mathrm{pp}}, T_{\mathrm{hh}}$ and $T_{\mathrm{ph}}$ for the case of two identical disks with $\mu=200 / a^{2}$ and $\Delta=50 / a^{2}$ and $\phi_{\Delta}=0$. The solid lines are the real parts of the corresponding quantities and the dashed lines the imaginary parts, respectively. Notice the difference in the magnitudes.

transparent expressions:

$$
\begin{aligned}
T_{\mathrm{ph}}= & \left(\sum_{m} t_{\mathrm{ph}}^{m}\right)^{2} \cos \phi_{\Delta} \equiv\left|T_{\mathrm{ph}}\right| \exp \left(i \phi_{\mathrm{ph}}\right) \cos \phi_{\Delta} \\
T_{\mathrm{pp}}= & \left(\sum_{m} t_{\mathrm{pp}}^{m}(-1)^{m}\right)^{2} \equiv\left|T_{\mathrm{pp}}\right| \exp \left(i \phi_{\mathrm{pp}}\right), \quad T_{\mathrm{hh}}=\left(\sum_{m} t_{\mathrm{hh}}^{m}(-1)^{m}\right)^{2} \equiv\left|T_{\mathrm{hh}}\right| \exp \left(i \phi_{\mathrm{hh}}\right) \\
\delta N(E) \approx & \frac{4\left|T_{\mathrm{ph}}\right| \cos \phi_{\Delta}}{\pi^{2} \sqrt{k_{\mathrm{h}} k_{\mathrm{p}}} R} \sin \left[\left(k_{\mathrm{p}}-k_{\mathrm{h}}\right) R+\phi_{\mathrm{ph}}\right]-\frac{2\left|T_{\mathrm{pp}}\right|}{\pi^{2} k_{\mathrm{p}} R} \cos \left(2 k_{\mathrm{p}} R+\phi_{\mathrm{pp}}\right) \\
& +\frac{2\left|T_{\mathrm{hh}}\right|}{\pi^{2} k_{\mathrm{h}} R} \cos \left(2 k_{\mathrm{h}} R-\phi_{\mathrm{hh}}\right)
\end{aligned}
$$

The structure functions $T_{\mathrm{ph}}, T_{\mathrm{pp}}$ and $T_{\mathrm{hh}}$ characterize the properties of the scatterers and are independent of the separation $R$ between them. In fig. 3 we show some typical behavior of these functions with the quasi-particle energy $E$. Note the great difference in size between the normal (pp, hh) and Andreev (ph) structure functions, especially when $E \leq \Delta$.

Even though these structure functions are relatively easy to evaluate numerically in the case of almost any conceivable size grains, it is extremely instructive to go one step further and derive an approximate expression for these structure functions themselves. An analysis of the structure functions (omitted here) eventually leads us to the following significantly simpler expression for the change in the density of states valid for $E \ll|\Delta|$ :

$$
\delta N(E)=-\operatorname{Im}\left\{\left[\int_{-a \min \left(k_{\mathrm{p}}, k_{\mathrm{h}}\right)}^{a \min \left(k_{\mathrm{p}}, k_{\mathrm{h}}\right)} \mathrm{d} \nu \exp \left(\frac{i\left(k_{\mathrm{p}}-k_{\mathrm{h}}\right) \nu^{2}}{2 k_{\mathrm{p}} k_{\mathrm{h}} a}\right)\right]^{2} \frac{e^{i\left(k_{\mathrm{p}}-k_{\mathrm{h}}\right)(R-2 a)}}{\pi^{2} R \sqrt{k_{\mathrm{p}} k_{\mathrm{h}}}}\right\} \cos \left(\phi_{\Delta}\right) .
$$

One can easily recognize the argument of the second exponential as the classical action of the shortest particle-hole periodic orbit. The hole momentum enters with a minus sign since it is anti-parallel to the hole velocity. The squared integral would become a product of similar factors in the case of two grains of different radii. For $E=0$ this overall factor becomes $4 m_{\max }^{2}=4 k_{\mathrm{F}}^{2} a^{2}$, i.e. the squared number of partial waves undergoing Andreev reflection. 


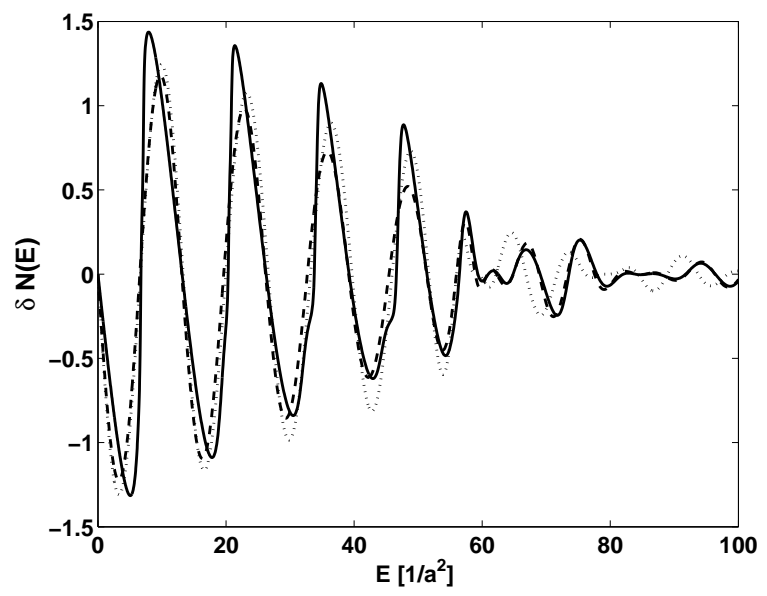

Fig. 4 - The density of states variation $\delta N(E)$ evaluated exactly (solid line), in the approximation of eq. (6) (dashed line) and under a further asymptotic expansion of the cylindrical functions in the structure functions (dotted line) for two identical disks with $R=6 a, \mu=200 / a^{2}, \Delta=50 / a^{2}$ and $\phi_{\Delta}=0$.

Results and conclusions. - In fig. [4 we compare the change in the density of states evaluated exactly (see eq. (5) ) with the simplified expression of eq. (6) and with a further asymptotic approximation of the structure functions on which eq. (7) is based. It is now obvious that the oscillatory character of the change in the density of states is fully accounted for by the shortest periodic orbit, quantized as expected by

$$
\left(k_{\mathrm{p}}-k_{\mathrm{h}}\right)(R-2 a)-2 \arccos (E / \Delta)=2 \pi n, \quad n=0, \pm 1, \pm 2, \cdots .
$$

The maxima in $\delta g(E)=\mathrm{d} \delta N(E) / \mathrm{d} E$ correspond exactly to those energies for which the above condition is fulfilled. At each Andreev reflection the reflected wave acquires an additional phase $\arccos (E / \Delta)$, leading to the overall minus sign in eq. (7), valid for the case $E \ll \Delta$.

By using the exact expression (5) or the simplifications (6) or (7), one can now easily evaluate the interaction energy of spherical superfluid grains in the Fermi sea,

$$
E_{\mathrm{SC}}(R)=\nu_{\mathrm{deg}} \int_{0}^{\infty} \mathrm{d} E E \frac{\mathrm{d} \delta N(E)}{\mathrm{d} E}=-\nu_{\mathrm{deg}} \int_{0}^{\infty} \mathrm{d} E \delta N(E),
$$

as function of their separation $R$. This Casimir energy is completely dominated by the states with $E \leq \Delta$. We thus obtain, by numerical integration, for two spherical three-dimensional grains

$$
E_{\mathrm{SC}}(R) \approx \nu_{\mathrm{deg}} \frac{\hbar^{2} k_{\mathrm{F}}^{2}}{2 m} \frac{k_{\mathrm{F}} a^{4} \cos \phi_{\Delta}}{2 \pi R^{2}(R-2 a)} X,
$$

where $X=\mathcal{O}(1)$ and positive and very weakly dependent on separation. By comparing with eq. (11), one can show that $E_{\mathrm{SC}}(R) \gg\left|E_{\mathrm{C}}(R)\right|$ for the typical case $k_{\mathrm{F}} a \gg 1$. This is easy to accept now: in the case of two superfluid grains, embedded in a normal Fermi system, there are many "bound states" (see fig. 2) due to the appearance of a large number of periodic orbits, whereas there exists only a single unstable periodic orbit between two hard spheres $[1,2]$.

In summary, we have constructed both exact and simplified expressions for the calculation of the density of states and the Fermionic Casimir energy in the novel case of two spherical superfluid scatterers immersed in a normal Fermi system. We have furthermore shown and 
explained that these quantities are significantly enhanced in comparison to the normal case where the obstacles are scatterers with hard-wall reflection conditions. Since the change in the density of states is dominated by the Andreev reflection, which is almost exactly backward, the case of many grains reduces simply to the sum over all unobstructed pairs of grains. In particular, one can expect that the energetics in portions of the neutron stars would be greatly affected when the Fermionic Casimir energy is taken into account - more so than was the case when only normal systems were considered $[1,8]$. Generalizations of the present results to more complicated geometries appear to be straightforward.

$$
* * *
$$

This work was supported in part by DOE grant DE-FG02-97ER41014, by the Polish Committee for Scientific Research (KBN) under Contract No. 1 P03B 05927 and by the Forschungszentrum Jülich under Contract No. 41445400 (COSY-067). We would like to thank one of the referees for valuable suggestion.

\section{REFERENCES}

[1] Bulgac A. And Magierski P., Nucl. Phys. A, 683 (2001) 695; Erratum: 703 (2002) 892; Phys. Scripta T, 90 (2001) 150.

[2] Bulgac A. And Wirzba A., Phys. Rev Lett., 87 (2001) 120404.

[3] Volovik G. E., JETP Lett., 73 (2001) 375; The Universe in a Helium Droplet, (Clarendon Press, Oxford) 2003, sect. 29-5.

[4] Andreev A. F., Zh. Eksp. Teor. Phys., 46 (1964) 1823 [JETP, 19 (1964) 1228]; de Gennes P. G., Superconductivity of Metals and Alloys (Addison-Wesley, Reading) 1998; Blonder G. E., Tinkham M. And Klapwijk T. M., Phys. Rev. B, 25 (1982) 4515.

[5] Feigel'man M. V. and Larkin A. I., Chem. Phys., 235 (1998) 107; Spivak B., Zyuzin A. And Hruska M., Phys. Rev. B, 64 (2001) 132502.

[6] Shytov A. V., Lee P. A. And Levitov L. S., Usp. Fiz. Nauk, 168 (1998) 222.

[7] Kosztin I., Maslov D. L. And Goldbart P. M., Phys. Rev. Lett., 75 (1995) 1735; Altland A. and Zirnbauer M. R., Phys. Rev. Lett., 76 (1996) 3420; Lodder A. And Nazarov Yu. V., Phys. Rev. B, 58 (1998) 5783; Schomerus H. and Beenakker C. W. J., Phys. Rev. Lett., 82 (1991) 2951; Ihra W. et al., Eur. Phys. J. B, 21 (2001) 425; Adagideli I. And Golddbart P. M., Int. J. Mod. Phys., 16 (2002) 1381; JaCQuod Ph. et al., Phys. Rev. Lett., 90 (2003) 207004; Kormányos A. et al., 70, 2004 (052512) and many other references therein.

[8] Bulgac A. et al., Tours Symposium on Nuclear Physics V, edited by M. ARnould et al., Vol. 704 (AIP Conference Proceedings, Melville, NY) 2004, pp. 483-487 and references therein.

[9] Sadzikowski M., Acta Phys. Polon. B, 33 (2002) 1601; Sadzikowski M. and Tachibana M., Phys.Rev. D, 66 (2002) 045024; Acta Phys. Polon. B, 33 (2002) 4141.

[10] Oaknin D. H. And Zhitnitsky A., Phys. Rev. D, 71 (2005) 023519.

[11] Henseler M., Wirzba A. And Guhr T., Ann. Phys. (N.Y.), 258 (1997) 286.

[12] Lloyd P., Proc. Phys. Soc., 90 (1967) 207; Lloyd P. And Smith P. V., Adv. Phys., 21 (1972) 69; Berry M. V., Ann. Phys. (N.Y.), 131 (1981) 163.

[13] Krein M. G., Math. Sborn., 33 (1953) 597; Sov. Math.-Dokl., 3 (1962) 707; Birman M. Sh. AND Krein M. G., Sov. Math.-Dokl., 3 (1962) 740; Thirring W., Quantum Mechanics of Atoms and Molecules (Springer-Verlag, New York) 1981, sect. 3.6; Birman M. Sh. And Yafaev D. R., St. Petersburg Math. J., 4 (1993) 833.

[14] Beth E. And Uhlenbeck G. E., Physica (Utrecht), 4 (1937) 915; Huang K., Statistical Mechanics (John Wiley \& Sons, New York) 1987, sect. 10.3. 